\title{
LI-RADS category 5 hepatocellular carcinoma: preoperative gadoxetic acid-enhanced MRI for early recurrence risk stratification after curative resection
}

\author{
Hong Wei $^{1} \cdot$ Hanyu Jiang ${ }^{1} \cdot$ Tianying Zheng ${ }^{1} \cdot$ Zhen Zhang $^{1} \cdot$ Caiwei Yang ${ }^{1} \cdot$ Zheng Ye $^{1} \cdot$ Ting Duan $^{1} \cdot$ Bin Song $^{1}$
}

Received: 27 March 2020 / Revised: 1 August 2020 / Accepted: 15 September 2020 / Published online: 1 October 2020

(C) The Author(s) 2020

\begin{abstract}
Objectives To explore the role of preoperative gadoxetic acid-enhanced MRI in stratifying the risk of early recurrence in patients with LR-5 hepatocellular carcinoma (HCC) by LI-RADS v2018 after curative resection.

Methods Between July 2015 and August 2018, this study evaluated consecutive treatment-naïve at-risk LR-5 HCC patients who underwent gadoxetic acid-enhanced MRI examination within 2 weeks before curative resection. The Cox regression analysis was performed to identify potential predictors of early recurrence. Disease-free survival (DFS) rates were analyzed and compared by using the Kaplan-Meier method and log-rank tests.

Results Fifty-three of 103 (51.5\%) patients experienced early recurrence. Three MRI findings were significantly associated with early recurrence: corona enhancement (hazard ratio [HR]: 2.116; $p=0.013$ ), peritumoral hypointensity on hepatobiliary phase (HBP) (HR: 2.262; $p=0.007$ ), and satellite nodule (HR: $2.777 ; p=0.005$ ). An additional risk factor was AFP level $>400 \mathrm{ng} / \mathrm{mL}$ (HR: $1.975 ; p=0.016$ ). Based on the number of MRI predictors, LR-5 HCC patients were stratified into three subgroups: LR-5a (60/103; no predictor), LR-5b (26/103; one predictor), and LR-5c (17/103; two or three predictors), with low, medium, and high risk of early recurrence, respectively. The 2-year DFS rate of LR-5a, LR-5b, and LR-5c patients was $65.0 \%, 38.5 \%$, and $5.9 \%$, respectively, while the corresponding median DFS was undefined, 17.1 months, and 5.1 months, respectively $(p<0.001)$.

Conclusions In at-risk LR-5 HCC patients, corona enhancement, peritumoral hypointensity on HBP, and satellite nodule could be used to preoperatively stratify the risk of early recurrence after hepatectomy.

Key Points

- Corona enhancement, peritumoral hypointensity on HBP, satellite nodule, and serum AFP level $>400 \mathrm{ng} / \mathrm{mL}$ were significant predictors of early recurrence in patients with LR-5 HCC after hepatectomy.

- Based on the number of predictive MRI findings, LR-5 HCC patients could be preoperatively stratified into three subgroups: $L R-5 a, L R-5 b$, and LR-5c, with significantly different risk of early recurrence and disease-free survival.

- Preoperative risk stratification is essential for the identification of patients at increased risk of postoperative early recurrence, which may contribute to risk-based personalized management for LR-5 HCC patients.
\end{abstract}

Keywords Carcinoma, hepatocellular $\cdot$ Liver $\cdot$ Prognosis $\cdot$ Magnetic resonance imaging $\cdot$ Gadoxetic acid

Hong Wei and Hanyu Jiang contributed equally to this work.

Electronic supplementary material The online version of this article (https://doi.org/10.1007/s00330-020-07303-9) contains supplementary material, which is available to authorized users.

Bin Song

songlab_radiology@163.com

1 Department of Radiology, West China Hospital of Sichuan University, No. 37 Guoxue Xiang, Chengdu 610041, Sichuan, China

$\begin{array}{ll}\text { Abbreviations } & \\ \text { AFP } & \text { Alpha-fetoprotein } \\ \text { CT } & \text { Computed tomography } \\ \text { DFS } & \text { Disease-free survival } \\ \text { HBP } & \text { Hepatobiliary phase } \\ \text { HCC } & \text { Hepatocellular carcinoma } \\ \text { LI-RADS/LR } & \text { Liver Imaging Reporting and Data System } \\ \text { LR } & \text { Likelihood ratio } \\ \text { M } & \text { Malignancy } \\ \text { MR } & \text { Magentic resonance } \\ \text { MRI } & \text { Magnetic resonance imaging }\end{array}$




$\begin{array}{ll}\text { MVI } & \text { Microvascular invasion } \\ \text { NC } & \text { Not categorizable } \\ \text { NPV } & \text { Negative predictive value } \\ \text { PPV } & \text { Positive predictive value } \\ \text { TIV } & \text { Tumor in vein }\end{array}$

\section{Introduction}

Hepatocellular carcinoma (HCC) is the most frequent primary liver malignancy and the third leading cause of cancer-related deaths [1]. Surgical resection is regarded as the first-line treatment option for HCC patients with well-preserved liver function [2]. Nevertheless, $\sim 70 \%$ of HCC patients develop tumor recurrence within 5 years after surgery [2]. Early recurrence (within 2 years after surgery) is predominantly attributable to the dissemination of the primary HCC and correlated with tumor-related factors (e.g., microvascular invasion, worse differentiation), whereas late recurrence (beyond 2 years after surgery) is more a result of new malignant clones and related to underlying liver conditions (e.g., liver cirrhosis) [2-6]. However, the risk factors of early recurrence, despite critical in terms of treatment, prognosis, and outcome, are mostly evaluated by postoperative pathologic examinations, and their utility in the preoperative context remains limited.

Imaging plays a vital role in the prognostic assessment of HCC patients. Emerging pieces of evidence indicated that preoperative magnetic resonance imaging (MRI) findings, such as rim enhancement, peritumoral hypointensity on hepatobiliary phase (HBP), and nonsmooth tumor margin, were independent risk factors of postoperative recurrence or microvascular invasion (MVI) - a potent risk factor of early recurrence of HCC [7-12]. Although promising, independent validations of these imaging features remain warranted.

Initially released in 2011, the Liver Imaging Reporting and Data System (LI-RADS) was developed to standardize the imaging diagnosis of HCC in at-risk patients [13]. According to the probability of HCC, LI-RADS assigns liver observations in high-risk patients with five major categories from definitely benign (LR-1) to definitely HCC (LR-5). Two additional categories, including LR-M and LR-TIV, are used to describe observations probably or definitely malignant but not HCC-specific and definite tumor in vein, respectively [14]. Among these categories, LR-5 can confirm the diagnosis of HCC with nearly $100 \%$ specificity [15], which can be treated without the need for pathologic confirmation. However, LR-5 HCCs represent a wide and heterogenous spectrum of tumors with distinct clinical outcomes, and not all LR-5 HCC patients with resectable tumors, those at increased risk of early recurrence, in particular, would benefit from curative resection. Considering that LI-RADS has been widely used in routine clinical practice, accurate preoperative risk stratification of early recurrence for LR-5 HCC patients is essential for clinical decision-making and patient management. To our knowledge, however, the utility of standardized imaging features defined by LI-RADS in predicting postoperative early recurrence and stratifying disease-free survival (DFS) of patients with LR-5 HCC has not been studied.

Therefore, the purpose of this study was to explore the role of preoperative gadoxetic acid-enhanced MRI features, particularly LI-RADS v2018 imaging features, in predicting early recurrence and stratifying DFS of patients with LR-5 HCC after curative resection.

\section{Materials and methods}

\section{Patients}

This single-center study used data from a prospectively collected observational cohort (Clinical trial registration no: ChiCTR1900026668) and was approved by our institutional review board. Written informed consent was waived. Between July 2015 and August 2018, 207 consecutive treatment-naïve patients with Child-Pugh class A at high-risk for developing HCC (i.e., those with cirrhosis or chronic hepatitis B virus infection) who underwent 3.0-T gadoxetic acid-enhanced MRI within 2 weeks before curative resection were included. Patients were excluded from the study if they (a) died of postoperative complications within 2 weeks $(n=1)$, (b) had ruptured HCCs $(n=1)$, (c) had a history of extrahepatic primary carcinoma $(n=6)$, (d) had non-HCC hepatic tumors confirmed by postoperative pathology $(n=40)$, (e) had incomplete pathology data $(n=1)$, (f) had no LR-5 observations $(n=23)$ or had LR-NC observations $(n=1)$ due to image omission or degradation in accordance with LI-RADS v2018 [14], and (g) were followed up for less than 2 years $(n=31)$ after surgery. Thus, our final study population consisted of 103 patients with pathologically confirmed LR-5 HCCs (Fig. 1).

Clinical information, laboratory data, and pathologic reports were retrieved from electronic medical records. All patients underwent curative resection (R0, defined as complete macroscopic removal of the tumor with a negative margin confirmed by histological examination) after adequate assessment of liver functional reserve, tumor extension, and patient will. The median time between MRI examination and surgery was 2 days (range, $0-12$ days).

\section{MRI examination}

All MRI examinations were performed by using a 3.0-T MRI system (Magnetom Skyra, Siemens Healthineers) equipped with an 18-channel body array coil. MRI sequences and parameters are listed in Supplementary Material 1. In- and opposed-phase images were initially obtained by using a T1weighted two-dimensional gradient-recalled-echo sequence. Dynamic images were obtained before and after contrast agent 


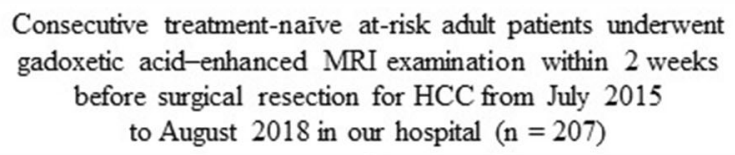

Patients died of postoperative complications within 2 weeks $(\mathrm{n}=1)$

Patients had ruptured $\mathrm{HCC}(\mathrm{n}=1)$

Patients had a history of extrahepatic primary carcinoma $(n=6)$

Postoperative pathologic analysis confirmed a diagnosis other than $\mathrm{HCC}(\mathrm{n}=40)$ : cholangiocarcinoma $(\mathrm{n}=21)$; combined $\mathrm{HCC}$ and cholangiocarcinoma $(n=4)$; other malignancies $(n=5)$; benign tumors and tumor-like lesions $(\mathrm{n}=10)$

Patients had incomplete pathology data $(n=1)$

Patients with pathologically-confirmed $\mathrm{HCC}(\mathrm{n}=158)$ ( $\mathrm{n}=10$ )

Patients had LR-NC observations due to image omission or degradation $(n=1)$

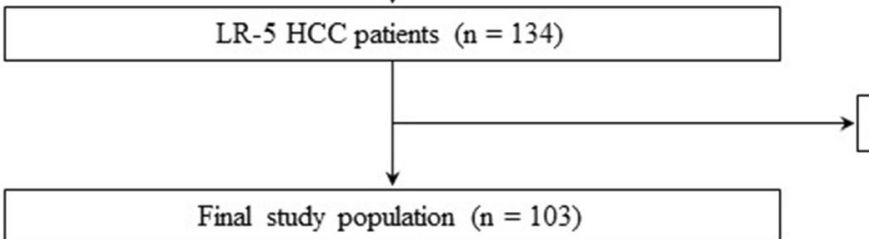

Patients were followed up for less than 2 years $(n=31)$

Fig. 1 Flow chart of the study population. HCC, hepatocellular carcinoma; NC, not categorizable

administration via a T1-weighted three-dimensional gradientecho sequence in the arterial phase (bolus triggering, $7 \mathrm{~s}$ after the peak enhancement of celiac trunk), portal venous phase (60$70 \mathrm{~s}$ after contrast agent injection), and transitional phase (180 s after contrast agent injection). For dynamic imaging, $0.1 \mathrm{~mL} / \mathrm{kg}$ $(0.025 \mathrm{mmol} / \mathrm{kg})$ of gadoxetic acid disodium (Primovist; Bayer Schering Pharma) was injected, followed by a $30 \mathrm{~mL}$ of $0.9 \%$ saline flush at a rate of $2.0 \mathrm{~mL} / \mathrm{s}$. Hepatobiliary phase (HBP) images were obtained $20 \mathrm{~min}$ after the injection of the contrast agent with the same sequence as that used for the pre- and postcontrast dynamic images. During the interval between transitional and HBP imaging, breath-hold fat-suppressed fast spinecho T2-weighted imaging was obtained. Diffusion-weighted images were also obtained by using a navigator-triggered technique at $b$ values of $0,50,500,800,1000$, and $1200 \mathrm{~s} / \mathrm{mm}^{2}$ and the corresponding apparent diffusion coefficient maps based on $b$ values 0 and $1200 \mathrm{~s} / \mathrm{mm}^{2}$ were reconstructed.

\section{Image analysis}

Preoperative MR images were retrieved from the Picture Archiving and Communication System and reviewed by two independent radiologists (J. H. Y. and Q.Y., both with 7 years of experience in abdominal MRI) who were blinded to the clinicopathologic and follow-up information. To resolve discrepancies between the two reviewers, all MR images were reassessed together with a third, more experienced radiologist (L.X.J., with 11 years of experience in abdominal MRI) until a consensus was reached.

For each patient at-risk for HCC, the imaging features and LI-RADS categories according to LI-RADS v2018 [14], along with the tumor size, number, and presence or absence of tumor in vein, were evaluated. The following non-LIRADS imaging features previously reported to be predictive of recurrence or MVI of HCC were also evaluated: nonsmooth tumor margin [12], peritumoral hypointensity on HBP [11], incomplete tumor capsule [16], and satellite nodule [17]. The definition of each assessed MR imaging feature is summarized in Supplementary Material 2.

HCC was considered single when nodules close to the primary tumor were designated as satellite nodules; otherwise, HCCs were considered multiple. For patients with multiple tumors, all measurable observations were assessed and the largest observation was selected as the representative for statistical analysis. 
Table 1 Clinicopathologic and MR imaging characteristics of the study population

\begin{tabular}{|c|c|c|c|c|}
\hline Characteristic & Total $^{*}(n=103)$ & Early recurrence absent* $(n=50)$ & Early recurrence present* $(n=53)$ & $p$ value \\
\hline Age (years) ${ }^{\dagger}$ & $51.0 \pm 12.0$ & $53.1 \pm 11.3$ & $49.0 \pm 12.4$ & 0.078 \\
\hline \multicolumn{4}{|l|}{ Sex } & \multirow[t]{3}{*}{0.559} \\
\hline Female & $21(20.4)$ & $9(18.0)$ & $12(22.6)$ & \\
\hline Male & $82(79.6)$ & $41(82.0)$ & $41(77.4)$ & \\
\hline \multicolumn{4}{|l|}{ HBsAg } & \multirow[t]{3}{*}{0.353} \\
\hline Negative & $17(16.5)$ & $10(20.0)$ & $7(13.2)$ & \\
\hline Positive & $86(83.5)$ & $40(80.0)$ & $46(86.8)$ & \\
\hline Serum ALT level (IU/L) $)^{\dagger}$ & $37.0(11.0-620.0)$ & $35.0(11.0-620.0)$ & $40.0(11.0-225.0)$ & 0.533 \\
\hline Serum AST level (IU/L) $)^{\dagger}$ & $39.0(18.0-243.0)$ & $36.5(19.0-176.0)$ & $42.0(18.0-243.0)$ & 0.438 \\
\hline Serum TBIL level $(\mu \mathrm{mol} / \mathrm{L})^{\dagger}$ & $13.9(4.2-54.2)$ & $13.7(7.7-54.2)$ & $14.6(4.2-31.1)$ & 0.642 \\
\hline Prothrombin time $(\mathrm{s})^{\dagger}$ & $12.3 \pm 1.0$ & $12.2 \pm 1.1$ & $12.3 \pm 1.0$ & 0.476 \\
\hline Platelet count $\left(\times 10^{\wedge} 9 / \mathrm{L}\right)^{\dagger}$ & $148.0(40.0-492.0)$ & $147.5(58.0-492.0)$ & $152.0(40.0-367.0)$ & 0.518 \\
\hline \multicolumn{4}{|l|}{ Serum AFP level } & \multirow[t]{3}{*}{0.026} \\
\hline$\leq 400 \mathrm{ng} / \mathrm{mL}$ & $65(63.1)$ & $37(74.0)$ & $28(52.8)$ & \\
\hline$>400 \mathrm{ng} / \mathrm{mL}$ & $38(36.9)$ & $13(26.0)$ & $25(47.2)$ & \\
\hline \multicolumn{4}{|l|}{ BCLC stage } & \multirow[t]{5}{*}{0.004} \\
\hline $0-\mathrm{A}$ & $35(34.0)$ & $23(46.0)$ & $12(22.6)$ & \\
\hline $\mathrm{AB}^{\dagger \dagger}$ & $38(36.9)$ & $18(36.0)$ & $20(37.7)$ & \\
\hline B & $8(7.8)$ & $3(6.0)$ & $5(9.4)$ & \\
\hline $\mathrm{C}$ & $22(21.4)$ & $6(12.0)$ & $16(30.2)$ & \\
\hline Pathologic features & & & & \\
\hline Tumor differentiation & & & & 0.022 \\
\hline Well or moderately differentiated & $54(52.4)$ & $32(64.0)$ & $22(41.5)$ & \\
\hline Poorly differentiated & 49 (47.6) & $18(36.0)$ & $31(58.5)$ & \\
\hline Microvascular invasion & & & & 0.042 \\
\hline Absent & $75(72.8)$ & $41(82.0)$ & $34(64.2)$ & \\
\hline Present & $28(27.2)$ & $9(18.0)$ & $19(35.8)$ & \\
\hline Serosal invasion & & & & 0.036 \\
\hline Absent & $55(53.4)$ & $32(64.0)$ & $23(43.4)$ & \\
\hline Present & 48 (46.6) & $18(36.0)$ & $30(56.6)$ & \\
\hline Satellite nodule & & & & 0.045 \\
\hline Absent & $94(91.3)$ & $49(98.0)$ & 45 (84.9) & \\
\hline Present & $9(8.7)$ & $1(2.0)$ & $8(15.1)$ & \\
\hline Cirrhosis & & & & 0.098 \\
\hline Absent & $64(62.1)$ & $27(54.0)$ & $37(69.8)$ & \\
\hline Present & $39(37.9)$ & $23(46.0)$ & $16(30.2)$ & \\
\hline MR imaging features & & & & \\
\hline Tumor size $(\mathrm{cm})^{\dagger}$ & $6.3 \pm 3.0$ & $5.4 \pm 2.5$ & $7.2 \pm 3.3$ & 0.002 \\
\hline No. of tumor & & & & 0.049 \\
\hline Single & $90(87.4)$ & $47(94.0)$ & $43(81.1)$ & \\
\hline Multiple & 13 (12.6) & $3(6.0)$ & $10(18.9)$ & \\
\hline LI-RADS major features & & & & \\
\hline Tumor size $\geq 20 \mathrm{~mm}$ & & & & 0.325 \\
\hline Absent & $5(4.9)$ & $4(8.0)$ & $1(1.9)$ & \\
\hline Present & $98(95.1)$ & $46(92.0)$ & $52(98.1)$ & \\
\hline Non-rim arterial phase hyperenhance & & & & $\ldots$ \\
\hline Absent & $0(0.0)$ & $0(0.0)$ & $0(0.0)$ & \\
\hline Present & $103(100.0)$ & $50(100.0)$ & $53(100.0)$ & \\
\hline Non-peripheral washout & & & & $\ldots$ \\
\hline Absent & $0(0.0)$ & $0(0.0)$ & $0(0.0)$ & \\
\hline Present & $103(100.0)$ & $50(100.0)$ & $53(100.0)$ & \\
\hline Enhancing capsule & & & & 0.205 \\
\hline Absent & $31(30.1)$ & $18(36.0)$ & $13(24.5)$ & \\
\hline Present & $72(69.9)$ & $32(64.0)$ & $40(75.5)$ & \\
\hline LI-RADS ancillary features (favoring & $\mathrm{C}$ in particular) & & & \\
\hline Non-enhancing capsule & & & & 0.055 \\
\hline Absent & $84(81.6)$ & $37(74.0)$ & $47(88.7)$ & \\
\hline Present & 19 (18.4) & $13(26.0)$ & $6(11.3)$ & \\
\hline Nodule-in-nodule architecture & & & & 0.099 \\
\hline Absent & $45(43.7)$ & $26(52.0)$ & $19(35.8)$ & \\
\hline Present & $58(56.3)$ & $24(48.0)$ & $34(64.2)$ & \\
\hline Mosaic architecture & & & & 0.280 \\
\hline Absent & $26(25.2)$ & $15(30.0)$ & $11(20.8)$ & \\
\hline Present & $77(74.8)$ & $35(70.0)$ & $42(79.2)$ & \\
\hline Fat in mass, more than adjacent liver & & & & 0.110 \\
\hline Absent & $81(78.6)$ & $36(72.0)$ & $45(84.9)$ & \\
\hline Present & $22(21.4)$ & $14(28.0)$ & $8(15.1)$ & \\
\hline Blood products in mass & & & & 0.197 \\
\hline Absent & $53(51.5)$ & $29(58.0)$ & $24(45.3)$ & \\
\hline Present & $50(48.5)$ & $21(42.0)$ & $29(54.7)$ & \\
\hline LI-RADS ancillary features (favoring & lignancy, not $\mathrm{HCC}$ in & ticular) & & \\
\hline Transitional phase hypointensity & & & & 1.000 \\
\hline Absent & $2(1.9)$ & $1(2.0)$ & $1(1.9)$ & \\
\hline Present & $101(98.1)$ & $49(98.0)$ & $52(98.1)$ & \\
\hline Restricted diffusion & & & & $\ldots$ \\
\hline Absent & $0(0.0)$ & $0(0.0)$ & $0(0.0)$ & \\
\hline Present & $103(100.0)$ & $50(100.0)$ & $53(100.0)$ & \\
\hline
\end{tabular}


Table 1 (continued)

\begin{tabular}{|c|c|c|c|c|}
\hline Characteristic & Total $^{*}(n=103)$ & Early recurrence absent* $(n=50)$ & Early recurrence present* $(n=53)$ & $p$ value \\
\hline \multicolumn{4}{|l|}{ Mild-moderate T2 hyperintensity } & \multirow{3}{*}{$\cdots$} \\
\hline Absent & $0(0.0)$ & $0(0.0)$ & $0(0.0)$ & \\
\hline Present & $103(100.0)$ & $50(100.0)$ & $53(100.0)$ & \\
\hline \multicolumn{4}{|l|}{ Corona enhancement } & \multirow[t]{3}{*}{0.001} \\
\hline Absent & $75(72.8)$ & $44(88.0)$ & $31(58.5)$ & \\
\hline Present & $28(27.2)$ & $6(12.0)$ & $22(41.5)$ & \\
\hline \multicolumn{4}{|l|}{ Fat sparing in solid mass } & \multirow[t]{3}{*}{0.262} \\
\hline Absent & $100(97.1)$ & $50(100.0)$ & $50(94.3)$ & \\
\hline Present & $3(2.9)$ & $0(0.0)$ & $3(5.7)$ & \\
\hline \multicolumn{4}{|l|}{ Hepatobiliary phase hypointensity } & \multirow[t]{3}{*}{0.569} \\
\hline Absent & $4(3.9)$ & $3(6.0)$ & $1(1.9)$ & \\
\hline Present & $99(96.1)$ & $47(94.0)$ & $52(98.1)$ & \\
\hline \multicolumn{4}{|l|}{ Iron sparing in solid mass } & \multirow[t]{3}{*}{0.485} \\
\hline Absent & $102(99.0)$ & $49(98.0)$ & $53(100.0)$ & \\
\hline Present & $1(1.0)$ & $1(2.0)$ & $0(0.0)$ & \\
\hline \multicolumn{4}{|l|}{ Tumor in vein } & \multirow[t]{3}{*}{0.024} \\
\hline Absent & $81(78.6)$ & $44(88.0)$ & $37(69.8)$ & \\
\hline Present & $22(21.4)$ & $6(12.0)$ & $16(30.2)$ & \\
\hline \multicolumn{5}{|l|}{ Non-LIRADS imaging features } \\
\hline \multicolumn{4}{|l|}{ Non-smooth tumor margin } & \multirow[t]{3}{*}{0.286} \\
\hline Absent & 48 (46.6) & $26(52.0)$ & $22(41.5)$ & \\
\hline Present & $55(53.4)$ & $24(48.0)$ & $31(58.5)$ & \\
\hline \multicolumn{4}{|l|}{ Peritumoral hypointensity on HBP } & \multirow[t]{3}{*}{0.002} \\
\hline Absent & 79 (76.7) & $45(90.0)$ & $34(64.2)$ & \\
\hline Present & $24(23.3)$ & $5(10.0)$ & $19(35.8)$ & \\
\hline \multicolumn{4}{|l|}{ Incomplete tumor capsule } & \multirow{3}{*}{0.205} \\
\hline Absent & $49(47.6)$ & $27(54.0)$ & $22(41.5)$ & \\
\hline Present & $54(52.4)$ & $23(46.0)$ & $31(58.5)$ & \\
\hline \multicolumn{4}{|l|}{ Satellite nodule } & \multirow[t]{2}{*}{0.003} \\
\hline Absent & $91(88.3)$ & $49(98.0)$ & $42(79.2)$ & \\
\hline Present & 12 (11.7) & $1(2.0)$ & $11(20.8)$ & 0.417 \\
\hline Single & $7(58.3)$ & $0(0.0)$ & $7(63.6)$ & 0.865 \\
\hline Multiple & $5(41.7)$ & $1(100.0)$ & $4(36.4)$ & \\
\hline Size $(\mathrm{cm})^{\dagger}$ & $1.4 \pm 0.4$ & 1.3 & $1.4 \pm 0.4$ & \\
\hline
\end{tabular}

$A F P$ alpha-fetoprotein, $A L T$ alanine aminotransferase, $A S T$ aspartate aminotransferase, $B C L C$ Barcelona Clinic Liver Cancer, $H B P$ hepatobiliary phase, $H C C$ hepatocellular carcinoma, LI-RADS Liver Imaging Reporting and Data System, TBIL total bilirubin

*Unless otherwise indicated, data are number of patients, with percentage in parentheses. Categorical variables were compared by using the chi-square test, Fisher exact test, or Kruskal-Wallis $H$ test

${ }^{\dagger}$ Data are continuous variables, reported as mean \pm standard deviation or median (range), and were compared by using the two-sample $t$ test or MannWhitney $U$ test

${ }^{\dagger} \mathrm{BCLC}$ stage $\mathrm{AB}$ was defined as a single large $(>5 \mathrm{~cm}) \mathrm{HCC}$ with Child-Pugh classification $\mathrm{A}-\mathrm{B}$ and performance status 0 and without vascular invasion

\section{Follow-up surveillance after surgical resection}

Postoperative follow-up was performed with contrastenhanced computed tomography (CT), MRI, or ultrasound, initially at 1 month after surgery and every 3 months thereafter. In addition, monthly blood tests for serum alphafetoprotein (AFP) level were obtained.

Early recurrence was defined as intrahepatic and/or extrahepatic recurrence within 2 years after resection of HCC. Recurrence was suspected when the serum AFP levels were highly elevated or new hepatic lesions were observed on ultrasound. Recurrence was established by radiologic evidence (CT or MRI) of new tumors. Increasing serum AFP levels alone without the evidence of new malignancies did not indicate recurrence. All patients were followed until death, early recurrence, or for at least 2 years after curative resection. DFS was defined as the interval from the date of surgery to that of tumor recurrence within 2 years (event), or to that of the last follow-up without early recurrence (censored).

\section{Statistical analysis}

Categorical variables, reported as absolute numbers and rates in percentages, were compared by using the chisquare test, Fisher exact test, or Kruskal-Wallis $H$ test, where applicable, whereas continuous variables, expressed as mean \pm standard deviation or median (range), were compared by using two-sample $t$ test or Mann-Whitney $U$ test, where applicable.

DFS was assessed and compared by using the Kaplan-Meier method and log-rank tests. A univariate Cox proportional hazards model was used to assess significant MR imaging findings and clinical factors associated with early recurrence. All variables with $p<0.1$ at univariate analysis were included for multivariate analysis using a stepwise Cox hazards regression model (forward LR). All statistical analyses were conducted by using SPSS software (version 22.0, IBM). A $p<0.05$ was considered statistically significant. 
Table 2 Univariate and multivariate analyses for early recurrence

\begin{tabular}{|c|c|c|c|c|c|c|}
\hline \multirow[b]{2}{*}{ Parameter } & \multicolumn{3}{|c|}{ Univariate analysis } & \multicolumn{3}{|c|}{ Multivariate analysis } \\
\hline & HR & $95 \% \mathrm{CI}$ & $p$ value & HR & $95 \% \mathrm{CI}$ & $p$ value \\
\hline Tumor size & 1.188 & $1.085,1.301$ & $<0.001$ & $\ldots$ & $\ldots$ & $\cdots$ \\
\hline Multiple tumors & 2.317 & $1.159,4.631$ & 0.017 & $\ldots$ & $\ldots$ & $\ldots$ \\
\hline \multicolumn{7}{|l|}{ LI-RADS major features } \\
\hline Tumor size $\geq 20 \mathrm{~mm}$ & 3.227 & $0.446,23.349$ & 0.246 & $\ldots$ & $\ldots$ & $\ldots$ \\
\hline Non-rim arterial phase hyperenhancement & $\ldots$ & $\ldots$ & $\cdots$ & $\cdots$ & $\cdots$ & $\cdots$ \\
\hline Non-peripheral washout & $\ldots$ & $\ldots$ & $\ldots$ & $\ldots$ & $\ldots$ & $\ldots$ \\
\hline Enhancing capsule & 1.382 & $0.739,2.584$ & 0.311 & $\ldots$ & $\ldots$ & $\ldots$ \\
\hline \multicolumn{7}{|c|}{ LI-RADS ancillary features (favoring HCC in particular) } \\
\hline Non-enhancing capsule & 0.475 & $0.203,1.112$ & 0.086 & $\ldots$ & $\ldots$ & $\ldots$ \\
\hline Nodule-in-nodule architecture & 1.637 & $0.933,2.873$ & 0.086 & $\ldots$ & $\ldots$ & $\ldots$ \\
\hline Mosaic architecture & 1.461 & $0.752,2.838$ & 0.264 & $\ldots$ & $\ldots$ & $\ldots$ \\
\hline Fat in mass, more than adjacent liver & 0.562 & $0.265,1.194$ & 0.134 & $\ldots$ & $\ldots$ & $\ldots$ \\
\hline Blood products in mass & 1.496 & $0.871,2.571$ & 0.145 & $\ldots$ & $\ldots$ & $\ldots$ \\
\hline \multicolumn{7}{|c|}{ LI-RADS ancillary features (favoring malignancy, not HCC in particular) } \\
\hline Transitional phase hypointensity & 1.162 & $0.161,8.413$ & 0.882 & $\ldots$ & $\ldots$ & $\ldots$ \\
\hline Restricted diffusion & $\ldots$ & $\ldots$ & $\ldots$ & $\ldots$ & $\ldots$ & $\ldots$ \\
\hline Mild-moderate T2 hyperintensity & $\ldots$ & $\ldots$ & $\ldots$ & $\ldots$ & $\ldots$ & $\ldots$ \\
\hline Corona enhancement & 2.880 & $1.656,5.010$ & $<0.001$ & 2.116 & $1.172,3.820$ & 0.013 \\
\hline Fat sparing in solid mass & 2.738 & $0.843,8.892$ & 0.094 & $\ldots$ & $\ldots$ & $\ldots$ \\
\hline Hepatobiliary phase hypointensity & 2.430 & $0.336,17.583$ & 0.379 & $\ldots$ & $\ldots$ & $\ldots$ \\
\hline Iron sparing in solid mass & 0.049 & $0.000,1631.454$ & 0.570 & $\ldots$ & $\ldots$ & $\ldots$ \\
\hline Tumor in vein & 2.332 & $1.290,4.217$ & 0.005 & $\ldots$ & $\ldots$ & $\ldots$ \\
\hline \multicolumn{7}{|l|}{ Non-LIRADS imaging features } \\
\hline Non-smooth tumor margin & 1.462 & $0.846,2.526$ & 0.174 & $\ldots$ & $\ldots$ & $\ldots$ \\
\hline Peritumoral hypointensity on HBP & 2.759 & $1.566,4.859$ & $<0.001$ & 2.262 & $1.248,4.100$ & 0.007 \\
\hline Incomplete tumor capsule & 1.442 & $0.834,2.492$ & 0.190 & $\ldots$ & $\ldots$ & $\ldots$ \\
\hline Satellite nodule & 3.490 & $1.764,6.905$ & $<0.001$ & 2.777 & $1.369,5.634$ & 0.005 \\
\hline \multicolumn{7}{|l|}{ Clinical factors } \\
\hline Serum AFP level $>400 \mathrm{ng} / \mathrm{mL}$ & 2.107 & $1.224,3.628$ & 0.007 & 1.975 & $1.136,3.437$ & 0.016 \\
\hline \multicolumn{7}{|l|}{ BCLC stage } \\
\hline $0-\mathrm{A}^{\dagger}$ & $\ldots$ & $\ldots$ & $\ldots$ & $\ldots$ & $\ldots$ & $\ldots$ \\
\hline $\mathrm{AB}^{\dagger \dagger}$ & 1.762 & $0.861,3.605$ & 0.121 & $\ldots$ & $\ldots$ & $\ldots$ \\
\hline B & 2.428 & $0.855,6.900$ & 0.096 & $\ldots$ & $\ldots$ & $\ldots$ \\
\hline $\mathrm{C}$ & 3.407 & $1.601,7.248$ & 0.001 & $\ldots$ & $\ldots$ & $\ldots$ \\
\hline
\end{tabular}

Variables with $p<0.1$ in univariate analysis were applied to multivariate analysis using a stepwise Cox proportional hazards regression model $A F P$ alpha-fetoprotein, BCLC Barcelona Clinic Liver Cancer, $C I$ confidence interval, $H B P$ hepatobiliary phase, $H C C$ hepatocellular carcinoma, $H R$ hazard ratio, LI-RADS Liver Imaging Reporting and Data System

${ }^{\dagger}$ Used as the reference category

${ }^{\dagger}$ BCLC stage $\mathrm{AB}$ was defined as a single large (>5 cm) $\mathrm{HCC}$ with Child-Pugh class A-B and performance status 0 and without vascular invasion

\section{Results}

\section{Baseline patient characteristics}

Key clinicopathologic and MR imaging characteristics of the study population are summarized in Table 1 . There were no significant differences between early recurrence present $(n=$ 53) and absent $(n=50)$ groups in terms of the most assessed characteristics. For clinical parameters, patients with early recurrence showed higher serum AFP levels $(p=0.026)$ and more advanced BCLC stages than those without early recurrence $(p=0.004)$. 
Table 3 Diagnostic performance of each significant factor in prediction of early recurrence

\begin{tabular}{|c|c|c|c|c|c|}
\hline Characteristic & Sensitivity (\%) & Specificity $(\%)$ & $\operatorname{PPV}(\%)$ & $\operatorname{NPV}(\%)$ & Accuracy $(\%)$ \\
\hline \multicolumn{6}{|l|}{ MR imaging finding } \\
\hline Corona enhancement & $41.5(22 / 53)$ & $88.0(44 / 50)$ & $78.6(22 / 28)$ & $58.7(44 / 75)$ & $64.1(66 / 103)$ \\
\hline Peritumoral hypointensity on HBP & $35.8(19 / 53)$ & $90.0(45 / 50)$ & $79.2(19 / 24)$ & $57.0(45 / 79)$ & $62.1(64 / 103)$ \\
\hline Satellite nodule & $20.8(11 / 53)$ & $98.0(49 / 50)$ & $91.7(11 / 12)$ & $53.8(49 / 91)$ & $58.3(60 / 103)$ \\
\hline \multicolumn{6}{|l|}{ Tumor marker } \\
\hline Serum AFP level > $400 \mathrm{ng} / \mathrm{mL}$ & $47.2(25 / 53)$ & $74.0(37 / 50)$ & $65.8(25 / 38)$ & $56.9(37 / 65)$ & $60.2(62 / 103)$ \\
\hline
\end{tabular}

$A F P$ alpha-fetoprotein, $H B P$ hepatobiliary phase, $M R$ magnetic resonance, $N P V$ negative predictive value, $P P V$ positive predictive value

With regard to pathologic features, patients with early recurrence had more poorly differentiated tumors $(58.5 \%$ vs. $36.0 \%, p=0.022)$, higher proportion of MVI $(35.8 \%$ vs. $18.0 \%, p=0.042)$, serosal invasion $(56.6 \%$ vs. $36.0 \%, p=0.036)$, and satellite nodule $(15.1 \%$ vs. $2.0 \%, p=0.045)$ than those without early recurrence.

Among MR imaging features, mean tumor size of patients with early recurrence was larger than that of patients without early recurrence $(7.2 \mathrm{~cm}$ vs. $5.4 \mathrm{~cm}, p=0.002)$. Multiple tumors $(18.9 \%$ vs. $6.0 \%, p=0.049)$, corona enhancement $(41.5 \%$ vs. $12.0 \%, p=0.001)$, tumor in vein $(30.2 \%$ vs. $12.0 \%, p=0.024)$, peritumoral hypointensity on HBP (35.8\% vs. $10.0 \%, p=0.002)$, and satellite nodule $(20.8 \%$ vs. $2.0 \%, p=0.003$ ) were significantly more frequent in patients with early recurrence compared with those without early recurrence.

\section{Early recurrence after curative resection}

During a median follow-up period of 22.1 months (range, 1.0 46.5 months), tumor early recurrence was observed in 51.5\% $(53 / 103)$ patients. Among patients with early recurrence, $83.0 \%(44 / 53)$ experienced intrahepatic recurrence, $7.5 \%$ (4/53) experienced extrahepatic recurrence, and the remaining 9.4\% (5/53) experienced combined intra- and extrahepatic recurrence. The distant metastasis sites included lungs $(n=$ $5)$, bone $(n=2)$, lymph nodes $(n=1)$, and adrenal glands $(n=1)$.

\section{Prediction of early recurrence}

Of 196 measurable HCC nodules, 158 (80.6\%) were categorized as LR-5. The two reviewers agreed on the LR-5 category of most cases (150 of 158 nodules, $94.9 \%$ ) and reached a

Table 4 Preoperative gadoxetic acid-enhanced MR imaging findings for predicting early recurrence after surgical resection of HCC in published studies

\begin{tabular}{|c|c|c|c|}
\hline Author & Year & $\begin{array}{l}\text { No. of } \\
\text { patients }\end{array}$ & Predictive MR imaging findings for early recurrence ${ }^{*}$ \\
\hline Ahn SJ et al [7] & 2019 & 179 & Satellite nodule, peritumoral hypointensity on HBP, absence of capsule, and texture parameter \\
\hline Lee $S$ et al $[8]$ & 2017 & 197 & Arterial peritumoral enhancement, non-smooth tumor margin, and peritumoral hypointensity on HBP \\
\hline An C et al [9] & 2015 & 268 & Rim enhancement, peritumoral parenchymal enhancement, satellite nodule, and tumor size \\
\hline $\begin{array}{l}\text { Arrizumi S et al } \\
{[12]^{\dagger}}\end{array}$ & 2011 & 61 & Non-smooth tumor margin on HBP \\
\hline Cha DI et al [18] & 2020 & 549 & $\begin{array}{l}\text { Arterial rim enhancement of the tumor, non-hypervascular hepatobiliary hypointense nodules, and tumor } \\
\text { size }\end{array}$ \\
\hline Zhang L et al $[19]^{\dagger}$ & 2019 & 82 & Corona enhancement and irregular tumor margin \\
\hline Zhang $\mathrm{Z}$ et al $[20]^{\dagger}$ & 2019 & 155 & Radiomics score, gross vascular invasion, and non-smooth tumor margin \\
\hline Kim S et al [21] & 2019 & 167 & Radiomics features with 3-mm border extension \\
\hline Hectors SJ et al $[22]^{\dagger}$ & 2020 & 48 & Texture features and tumor size \\
\hline $\begin{array}{l}\text { Wei H et al } \\
\text { (this study) }\end{array}$ & 2020 & 103 & Corona enhancement, peritumoral hypointensity on HBP, and satellite nodule \\
\hline
\end{tabular}

$H B P$ hepatobiliary phase, $H C C$ hepatocellular carcinoma, $M R$ magnetic resonance

*Unless otherwise indicated, early recurrence was defined as recurrence within 2 years after resection of HCC

${ }^{\dagger}$ Early recurrence was defined as recurrence within 1 year after resection of HCC 


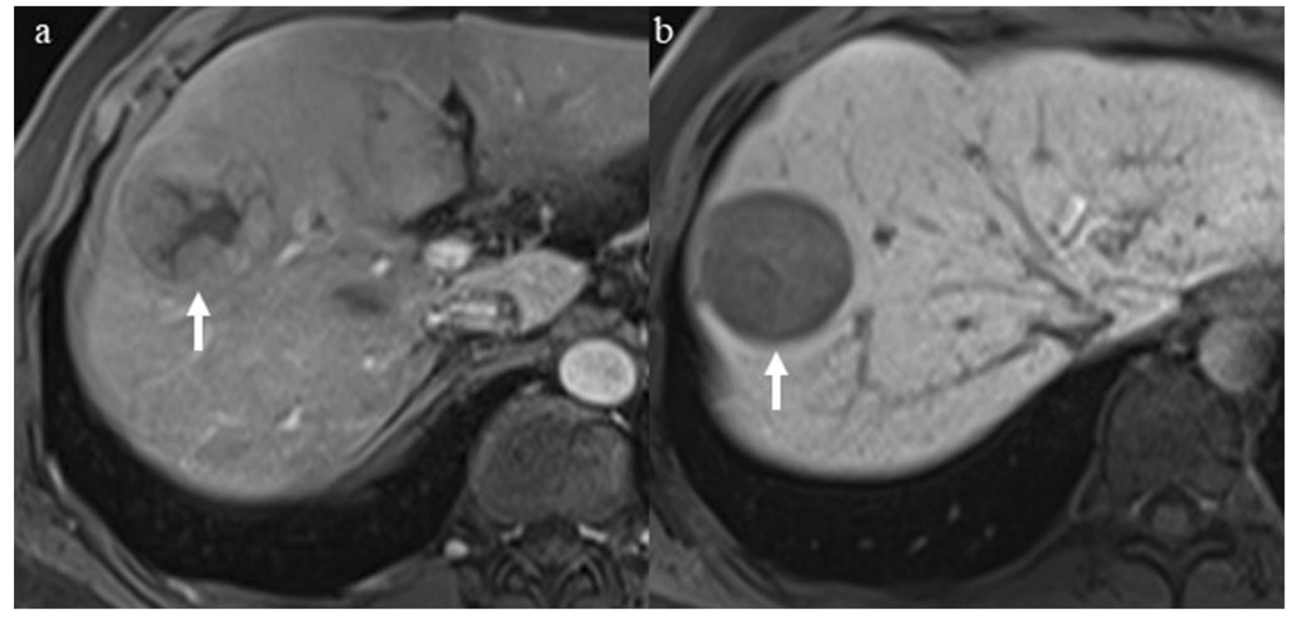

Fig. 2 A 47-year-old man with a 4.5-cm moderately differentiated LR-5 HCC in hepatic segment VIII and the serum AFP level was higher than $1210 \mathrm{ng} / \mathrm{mL}$. a Mass shows non-rim hyperenhancement and mosaic architecture (arrow) on late arterial phase. b Mass shows hypointensity and non-smooth tumor margin (arrow) on hepatobiliary phase. This patient had none of the predictive MR imaging findings for early recurrence and was categorized as LR-5a. There was no early recurrence during followup after curative resection. The disease-free survival was 37.6 months consensus through reassessment and discussion of the remaining 8 nodules with the third reviewer.

Tumor size, multiple tumors, non-enhancing capsule, nodule-in-nodule architecture, corona enhancement, fat sparing in solid mass, tumor in vein, peritumoral hypointensity on HBP, satellite nodule, serum AFP level $>400 \mathrm{ng} / \mathrm{mL}$, and BCLC stage were identified as significant predictors of early recurrence at univariate analysis. However, only corona enhancement (hazard ratio [HR]: $2.116 ; p=0.013$ ), peritumoral hypointensity on HBP (HR: $2.262 ; p=0.007$ ), satellite nodule (HR: 2.777; $p=0.005$ ), and serum AFP level $>400 \mathrm{ng} / \mathrm{mL}$ (HR: $1.975 ; p=0.016$ ) were significant predictors of early recurrence at multivariate analysis (Table 2).

The sensitivity, specificity, PPV, NPV, and accuracy of each indicator predictive of early recurrence are summarized in Table 3. In addition, preoperative gadoxetic acid-enhanced MR imaging features for predicting early recurrence of $\mathrm{HCC}$ after surgical resection in published studies [7-9, 12, 18-22] are shown in Table 4.

\section{Imaging features for DFS stratification}

Based on the number of MR imaging predictors, 103 LR-5 HCC patients were stratified into three subgroups according to their risk of early recurrence: LR-5a (low-risk patients, 60/ 103), LR-5b (medium-risk patients, 26/103), and LR-5c (high-risk patients, 17/103) with no, one, or no less than two imaging predictors of early recurrence, respectively (Figs. 2, 3 , and 4). The 2-year DFS rate of LR-5a, LR-5b, and LR-5c HCC patients was $65.0 \%$ (95\% confidence interval [CI]: $54.0 \%, 78.3 \%$ ), 38.5\% (95\% CI: $23.7 \%, 62.5 \%$ ), and $5.9 \%$ (95\% CI: $0.9 \%, 39.4 \%)$, respectively $(p<0.001)$; while the corresponding median DFS was undefined, 17.1 months
(95\% CI: 4.9 months, 29.3 months) and 5.1 months (95\% CI: 3.7 months, 6.5 months), respectively $(p<0.001)$ (Fig. 5).

Furthermore, for LR-5a, LR-5b, and LR-5c subgroups, patients with serum AFP level $>400 \mathrm{ng} / \mathrm{mL}$ had lower 2-year DFS rate than those with serum AFP level $\leq 400 \mathrm{ng} / \mathrm{mL}$, but the differences were not significant (LR-5a: $55.0 \%$ (95\% CI: $37.0 \%, 81.8 \%$ ) vs. $70.0 \%$ (95\% CI: $57.1 \%, 85.7 \%), p=0.200$; LR-5b: $25.0 \%$ (95\% CI: $7.5 \%, 83.0 \%)$ vs. $44.4 \%$ (95\% CI: $26.5 \%, 74.5 \%), p=0.091$; LR-5c: $0.0 \%$ vs. $14.3 \%$ (95\% CI: $2.3 \%, 87.7 \%$ ), $p=0.639$ ) (Fig. 6).

\section{Pathologic features in relation to predictive MR imaging findings}

Pathologic features in relation to predictive MR imaging findings are shown in Table 5. HCCs with corona enhancement showed worse tumor differentiation than those without this finding $(67.9 \%$ vs. $40.0 \%$ poorly differentiated tumors, $p=$ 0.012). HCCs with peritumoral hypointensity on HBP showed a significantly higher proportion of MVI compared with those without this finding ( $50.0 \%$ vs. $20.3 \%, p=0.004)$. HCCs with satellite nodule on MRI showed a significantly higher proportion of pathologically determined satellite nodule than those without this finding $(41.7 \%$ vs. $4.4 \%, p<0.001)$.

\section{Discussion}

The results of this study demonstrated that based on the number of three MR imaging findings (corona enhancement, peritumoral hypointensity on HBP, and satellite nodule), LR-5 HCC patients could be preoperatively stratified into 

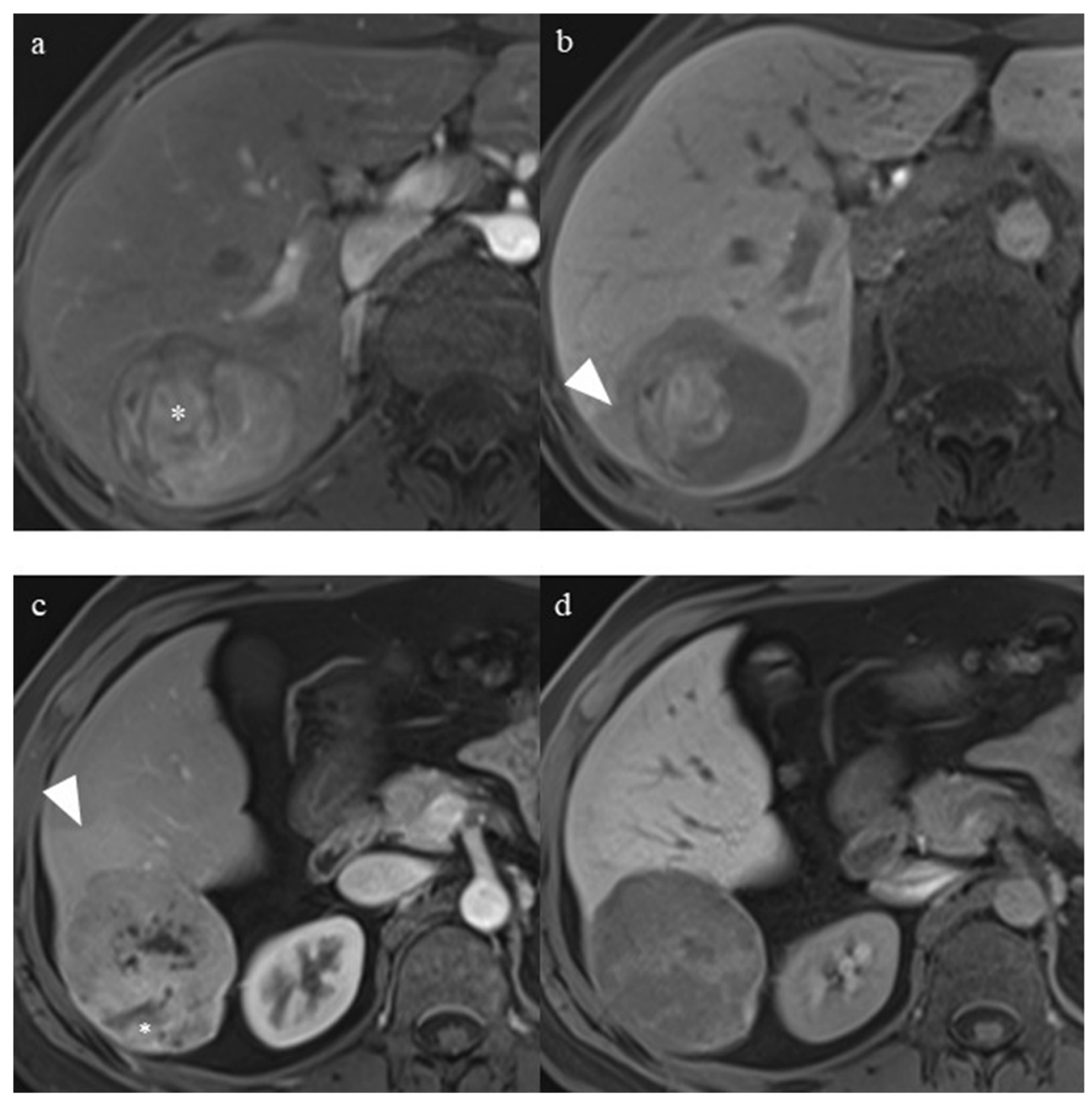

Fig. 3 a, b A 47-year-old woman with a 5.2-cm poorly differentiated LR$5 \mathrm{HCC}$ in hepatic segment VI and serum AFP level of $1.38 \mathrm{ng} / \mathrm{mL}$. a Mass shows non-rim hyperenhancement, nodule-in-nodule (asterisk), and mosaic architecture on late arterial phase. b Mass shows mixed intensity and peritumoral hypointensity (arrowhead) on hepatobiliary phase (HBP). This patient had one of the predictive MR imaging findings (peritumoral hypointensity on HBP) for early recurrence and was categorized as LR-5b. Early recurrence occurred in the liver during follow-up after curative resection. The disease-free survival was 23.7 months. c, d A 29 -year-old man with a $7.0-\mathrm{cm}$ moderately differentiated LR-5 HCC in

three subgroups: LR-5a, LR-5b, and LR-5c, with significantly different risk of early recurrence. This could be of clinical significance, considering that LR-5 HCC can be treated without the requirement for histologic confirmation. Preoperative risk stratification is essential for clinicians to identify patients at increased risk of postoperative early recurrence, which may contribute to risk-based personalized management, such as considering the use of extended resection or liver transplantation for selected LR-5c HCC patients, or making tailored follow-up plans and subsequent adjuvant treatment strategies for them.

As opposed to most previous studies in which patients were classified into two subgroups (low and high risk) according to their risk of recurrence [7, 9, 10, 18-22], herein, we identified hepatic segment VI and the serum AFP level was higher than $1210 \mathrm{ng}$ $\mathrm{mL}$. c Mass shows non-rim hyperenhancement, nodule-in-nodule (asterisk), mosaic architecture, and corona enhancement (arrowhead) on late arterial phase. d Mass shows hypointensity and non-smooth tumor margin on hepatobiliary phase. This patient had one of the predictive MR imaging findings (corona enhancement) for early recurrence and was categorized as LR-5b. Early recurrence occurred in the liver during follow-up after curative resection. The disease-free survival was 8.5 months

a fraction of medium-risk patients by stratifying LR-5 HCC patients into three subcategories with significantly different early recurrence rate and disease-free survival. Although Qin $\mathrm{X}$ et al stratified HCC patients into three subgroups with low, medium, and high recurrence rate by using the parameters of T1 mapping, the recurrence rates were not significantly different between subgroups of high- and medium-risk of recurrence in that study [23]. In addition, our method only requires counting the number of the risk factors, with complicated equations or calculations uninvolved, which enables clinicians to estimate the risk stratification quickly and discuss with patients efficiently before treatment.

Corona enhancement and satellite nodule were significant MR imaging findings for predicting early recurrence of $\mathrm{HCC}$ 
Fig. 4 A 38-year-old man with a $10.0-\mathrm{cm}$ poorly differentiated LR5 HCC in hepatic segment V-VIII and serum AFP level of $5.06 \mathrm{ng} /$ $\mathrm{mL}$. a Mass shows non-rim hyperenhancement, nodule-innodule (asterisk), and mosaic architecture on late arterial phase. b Mass shows hypointensity, nonsmooth tumor margin, and peritumoral hypointensity (arrowhead) on hepatobiliary phase. c A $1.7-\mathrm{cm}$ satellite nodule (arrow) located in the peritumoral parenchyma shows non-rim hyperenhancement on late arterial phase. d The satellite nodule (arrow) shows non-peripheral "washout" on portal venous phase. This patient had two of the predictive MR imaging findings (peritumoral hypointensity on HBP and satellite nodule) for early recurrence and was categorized as LR-5c. Early recurrence occurred in the liver during follow-up after curative resection. The disease-free survival was 3.7 months

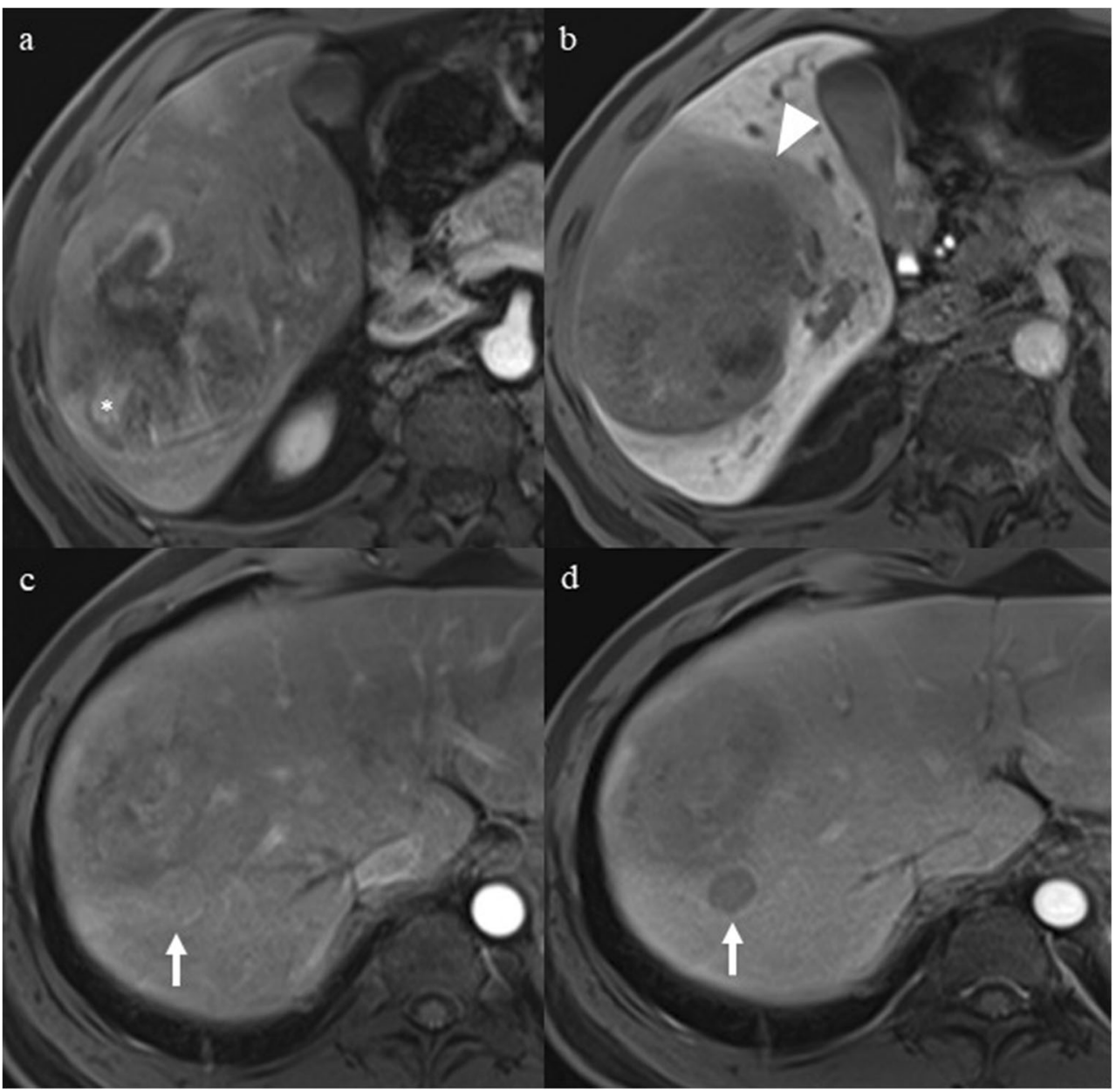

in our study. These results corresponded well with several previous studies $[7,9,19]$. Prior findings suggested that the presence of corona enhancement could indicate the progressed HCC that has a propensity to invade drainage vessels of the tumor, leading to intrahepatic metastases [24-26]. These metastases usually manifested as satellite nodules within the venous drainage area around the tumor, which can lead to vascular invasion and metastases [25, 27]. Likewise, our study also showed that HCCs with corona enhancement had worse tumor differentiation than those without this finding, which is supported by these studies.

Peritumoral hypointensity on HBP was another MR imaging finding predictive of early recurrence of $\mathrm{HCC}$ in our study, consistent with previous studies [7, 8]. Peritumoral hypointensity on HBP has been reported as a preoperative biomarker for MVI, a well-validated risk factor of early recurrence of HCC $[8,11]$. Similarly, in our study, among three predictive MR imaging findings, MVI is more frequently observed only in HCCs with peritumoral hypointensity on HBP than those without this finding, as with these studies. One possible mechanism underlying the appearance of peritumoral hypointensity on HBP is that peritumoral perfusion changes due to MVI might influence the function of organic aniontransporting polypeptide transporters, leading to a decrease in gadoxetic acid uptake in peritumoral hepatocytes [8]. However, in a small fraction of patients in our study, peritumoral hypointensity on HBP was also accompanied by the presence of hypointense areas in the hepatic segment where the tumors were located. In these cases, we speculated that other potential causes, such as biliary stasis, may have resulted in the appearance of hypointensity in the hepatic segment adjacent to the tumors on HBP. In this context, it might be challenging to determine the exact cause of peritumoral hypointensity on HBP. Currently, few published studies have adequately explored the pathologic causes of peritumoral hypointensity on HBP in HCCs. Further studies looking at this issue are required.

Serum AFP level $>400 \mathrm{ng} / \mathrm{mL}$ was an additional significant factor for predicting early recurrence in our study. Zhang $\mathrm{Y}$ et al have reported that serum AFP level $>400 \mathrm{ng} / \mathrm{mL}$ was related to HCC recurrence after hepatectomy [28], consistent with our result. Nevertheless, in LR-5a, LR-5b, and LR-5c subgroups, the 2-year DFS rates were not significantly different between patients with serum AFP level $>400 \mathrm{ng} / \mathrm{mL}$ and 
Fig. 5 Disease-free survival (DFS) curves of LR-5a (green), LR-5b (blue), and LR-5c (red) subgroups, with LR-5 HCC patients having no, one, and two or three significant MR imaging findings for predicting early recurrence, respectively. DFS differed significantly among three subgroups of LR-5 HCC patients according to the Kaplan-Meier method and log-rank tests

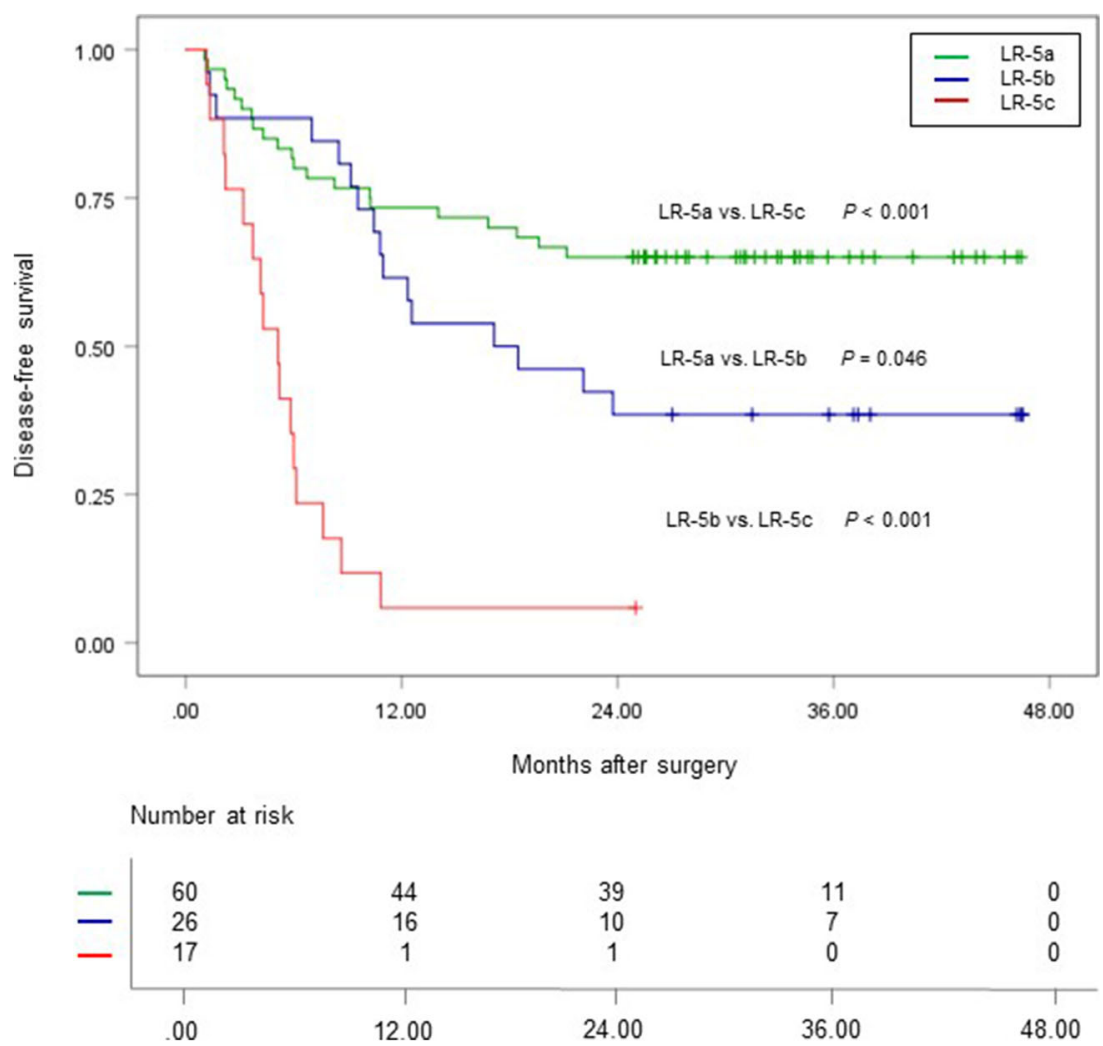

those with serum AFP level $\leq 400 \mathrm{ng} / \mathrm{mL}$. This indicates that MR imaging findings are superior to the serum AFP level in predicting early recurrence of HCC.
The LI-RADS major features (except for threshold growth) and ancillary features (except for corona enhancement), and other non-LIRADS imaging features reported to be correlated

Table 5 Pathologic features in relation to predictive MR imaging findings

\begin{tabular}{|c|c|c|c|c|c|c|c|c|c|}
\hline \multirow[t]{2}{*}{ Pathologic feature } & \multicolumn{3}{|c|}{ Corona enhancement } & \multicolumn{3}{|c|}{ Peritumoral hypointensity on HBP } & \multicolumn{3}{|c|}{ Satellite nodule } \\
\hline & Absent & Present & $p$ & Absent & Present & $p$ & Absent & Present & $p$ \\
\hline Tumor differentiation & & & 0.012 & & & 0.095 & & & 0.858 \\
\hline Well or moderately differentiated & $45(60.0)$ & $9(32.1)$ & & $45(57.0)$ & $9(37.5)$ & & $48(52.7)$ & $6(50.0)$ & \\
\hline Poorly differentiated & $30(40.0)$ & $19(67.9)$ & & $34(43.0)$ & $15(62.5)$ & & $43(47.3)$ & $6(50.0)$ & \\
\hline Microvascular invasion & & & 0.490 & & & 0.004 & & & 0.870 \\
\hline Absent & $56(74.7)$ & $19(67.9)$ & & $63(79.7)$ & $12(50.0)$ & & $67(73.6)$ & $8(66.7)$ & \\
\hline Present & $19(25.3)$ & $9(32.1)$ & & $16(20.3)$ & $12(50.0)$ & & $24(26.4)$ & $4(33.3)$ & \\
\hline Serosal invasion & & & 0.190 & & & 0.703 & & & 0.386 \\
\hline Absent & $43(57.3)$ & $12(42.9)$ & & $43(54.4)$ & $12(50.0)$ & & $50(54.9)$ & $5(41.7)$ & \\
\hline Present & $32(42.7)$ & $16(57.1)$ & & $36(45.6)$ & $12(50.0)$ & & $41(45.1)$ & $7(58.3)$ & \\
\hline Satellite nodule & & & 0.107 & & & 0.247 & & & $<0.001$ \\
\hline Absent & $71(94.7)$ & $23(82.1)$ & & 74 (93.7) & $20(83.3)$ & & 87 (95.6) & $7(58.3)$ & \\
\hline Present & $4(5.3)$ & $5(17.9)$ & & $5(6.3)$ & $4(16.7)$ & & $4(4.4)$ & $5(41.7)$ & \\
\hline Cirrhosis & & & 0.465 & & & 0.050 & & & 0.978 \\
\hline Absent & $45(60.0)$ & $19(67.9)$ & & $45(57.0)$ & $19(79.2)$ & & $56(61.5)$ & $8(66.7)$ & \\
\hline Present & $30(40.0)$ & $9(32.1)$ & & $34(43.0)$ & $5(20.8)$ & & $35(38.5)$ & $4(33.3)$ & \\
\hline
\end{tabular}

Data are number of patients with percentage in parentheses

$H B P$ hepatobiliary phase, $M R$ magnetic resonance 

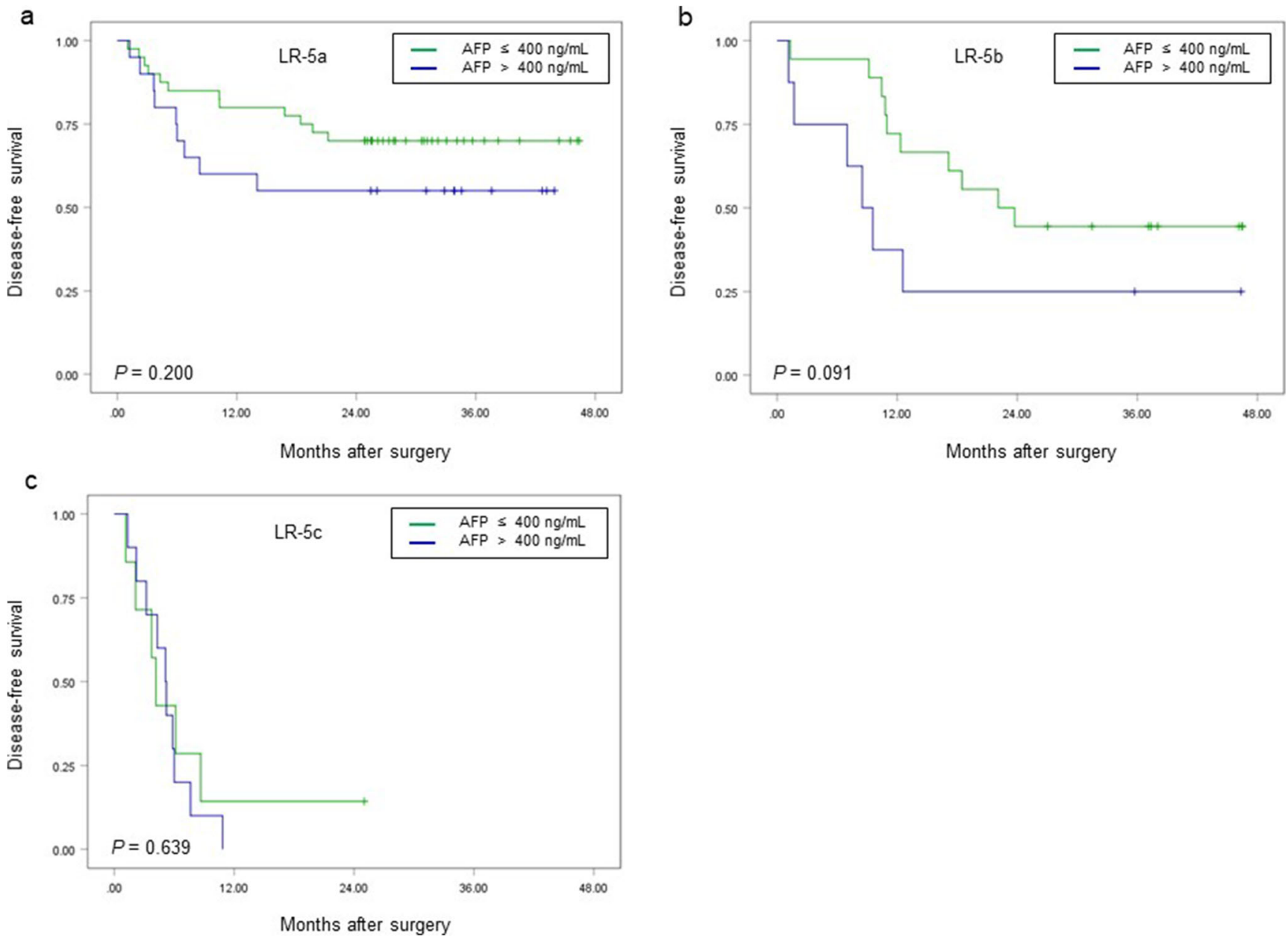

Fig. 6 Comparison of disease-free survival (DFS) according to the serum AFP level in LR-5a (a), LR-5b (b), and LR-5c (c) subgroups. There were no significant differences in DFS between patients with serum AFP level $>400 \mathrm{ng} / \mathrm{mL}$ and those with serum AFP level $\leq 400 \mathrm{ng} / \mathrm{mL}$ for three subgroups

with postoperative recurrence, such as tumor size [9, 18, 22], multiple tumors $[10,29,30]$, non-smooth tumor margin [8, $10,12,19,20]$, and incomplete tumor capsule [31], did not show significant associations with early recurrence in our study. However, as reported in a recent study, the absence of fat in mass (one LI-RADS ancillary features favoring HCC in particular) was an independent predictor for recurrence in patients with LR-5 HCC [10]. This may be partly due to the different endpoint events between our study and the previous study, in which recurrent HCCs were not distinguished as early and late recurrence. In addition, the different study populations (only LR-5 HCCs were enrolled in our study) and the small proportion of multiple HCCs (12.6\%) may be partly responsible for the discrepant results between our study and the prior studies.

Counterintuitively, in our study, tumor in vein (TIV) was not a significant predictor of early recurrence at multivariate analysis. A possible explanation may be that our study population were all selected surgical candidates with resectable tumors. In other words, patients with TIV involving the major blood vessels were excluded. Thus, the less severe TIV of our study population may be inadequate to result in the increased risk of early recurrence.

Intriguingly, BCLC stage was not independently associated with early recurrence in our study. This indicates that the designation of BCLC stage B or C should not be an absolute contraindication for surgery. Surgical resection could be considered for selected patients with BCLC stage B or C after careful assessment of liver functional remnant, tumor expansion, and patient will. However, our findings remain to be validated further since the relationship between BCLC stage and late recurrence was not estimated in our study, and there was a relatively small proportion of BCLC stage B and C patients $(29.1 \%, 30 / 103)$ in our study population.

This study had several limitations. First, substantial selection bias could have been introduced due to the retrospective nature, together with the limited sample size. Future studies including prospective multicenter enrollment will be required to validate the results of our study. Second, we only assessed LR-5 observations that were pathologically confirmed as 
HCC, whereas non-HCC LR-5 observations were not included. However, the pooled percentage of HCC for LR- 5 observation has been reported to be $94 \%$ [32], suggesting that nonHCC LR-5 observation is infrequent. Finally, in this study, the follow-up time was relatively short and the late recurrence was not assessed. Future studies with longer follow-up period are warranted to evaluate the role of MR imaging features in predicting both early and late HCC recurrence and stratifying long-term survival time.

In conclusion, in at-risk patients with LR-5 HCC, corona enhancement, peritumoral hypointensity on HBP, and satellite nodule can be used to effectively predict early recurrence and preoperatively stratify the DFS after curative resection. This may contribute to the risk-based personalized management for LR-5 HCC patients.

Acknowledgments We thank Dr. Yun Qin and Dr. Xijiao Liu from West China Hospital of Sichuan University for their help with the assessment of MR images.

Funding This work was supported by the National Natural Science Foundation of China (No. 81971571) and the 1.3 .5 project for disciplines of excellence, West China Hospital, Sichuan University (ZYJC18008).

\section{Compliance with ethical standards}

Guarantor The scientific guarantor of this publication is Bin Song.

Conflict of interest The authors of this manuscript declare no relationships with any companies whose products or services may be related to the subject matter of the article.

Statistics and biometry No complex statistical methods were necessary for this paper.

Informed consent Written informed consent was waived by the Institutional Review Board.

Ethical approval Institutional Review Board approval was obtained.

\author{
Methodology \\ - retrospective \\ - diagnostic or prognostic study \\ - performed at one institution
}

Open Access This article is licensed under a Creative Commons Attribution 4.0 International License, which permits use, sharing, adaptation, distribution and reproduction in any medium or format, as long as you give appropriate credit to the original author(s) and the source, provide a link to the Creative Commons licence, and indicate if changes were made. The images or other third party material in this article are included in the article's Creative Commons licence, unless indicated otherwise in a credit line to the material. If material is not included in the article's Creative Commons licence and your intended use is not permitted by statutory regulation or exceeds the permitted use, you will need to obtain permission directly from the copyright holder. To view a copy of this licence, visit http://creativecommons.org/licenses/by/4.0/.

\section{References}

1. Forner A, Reig M, Bruix J (2011) Hepatocellular carcinoma. Lancet 391:1301-1314

2. Vilarinho S, Calvisi DF (2014) New advances in precision medicine for hepatocellular carcinoma recurrence prediction and treatment. Hepatology 60:1812-1814

3. Portolani N, Coniglio A, Ghidoni S et al (2006) Early and late recurrence after liver resection for hepatocellular carcinoma: prognostic and therapeutic implications. Ann Surg 243:229-235

4. Poon RT, Fan ST, Ng IO, Lo CM, Liu CL, Wong J (2000) Different risk factors and prognosis for early and late intrahepatic recurrence after resection of hepatocellular carcinoma. Cancer 89:500-507

5. Imamura H, Matsuyama Y, Tanaka E et al (2003) Risk factors contributing to early and late phase intrahepatic recurrence of hepatocellular carcinoma after hepatectomy. J Hepatol 38:200-207

6. Cucchetti A, Piscaglia F, Caturelli E et al (2009) Comparison of recurrence of hepatocellular carcinoma after resection in patients with cirrhosis to its occurrence in a surveilled cirrhotic population. Ann Surg Oncol 16:413-422

7. Ahn SJ, Kim JH, Park SJ, Kim ST, Han JK (2019) Hepatocellular carcinoma: preoperative gadoxetic acid-enhanced MR imaging can predict early recurrence after curative resection using image features and texture analysis. Abdom Radiol (NY) 44:539-548

8. Lee S, Kim SH, Lee JE, Sinn DH, Park CK (2017) Preoperative gadoxetic acid-enhanced MRI for predicting microvascular invasion in patients with single hepatocellular carcinoma. J Hepatol 67:526-534

9. An C, Kim DW, Park YN, Chung YE, Rhee H, Kim MJ (2015) Single hepatocellular carcinoma: preoperative MR imaging to predict early recurrence after curative resection. Radiology 276:433443

10. Chen J, Zhou J, Kuang S et al (2019) Liver imaging reporting and data system category 5: MRI predictors of microvascular invasion and recurrence after hepatectomy for hepatocellular carcinoma. AJR Am J Roentgenol 213:821-830

11. Kim KA, Kim MJ, Jeon HM et al (2012) Prediction of microvascular invasion of hepatocellular carcinoma: usefulness of peritumoral hypointensity seen on gadoxetate disodium-enhanced hepatobiliary phase images. J Magn Reson Imaging 35:629-634

12. Ariizumi S, Kitagawa K, Kotera $Y$ et al (2011) A non-smooth tumor margin in the hepatobiliary phase of gadoxetic acid disodium (Gd-EOB-DTPA)-enhanced magnetic resonance imaging predicts microscopic portal vein invasion, intrahepatic metastasis, and early recurrence after hepatectomy in patients with hepatocellular carcinoma. J Hepatobiliary Pancreat Sci 18:575-585

13. Chernyak V, Fowler KJ, Kamaya A et al (2018) Liver imaging reporting and data system (LI-RADS) version 2018: imaging of hepatocellular carcinoma in at-risk patients. Radiology 289:816830

14. American College of Radiology (2018) CT/MRI liver imaging reporting and data system v2018 core. American College of Radiology, America Available via https:/www.acr.org/ClinicalResources/Reporting-and-Data-Systems/LI-RADS/CT-MRI-LIRADS-v2018. Accessed 28 Dec 2019

15. Kim DH, Choi SH, Park SH et al (2019) Meta-analysis of the accuracy of liver imaging reporting and data system category 4 or 5 for diagnosing hepatocellular carcinoma. Gut 68:1719-1721

16. Kim H, Park MS, Choi JY et al (2009) Can microvessel invasion of hepatocellular carcinoma be predicted by pre-operative MRI? Eur Radiol 19:1744-1751

17. Roayaie S, Blume IN, Thung SN et al (2009) A system of classifying microvascular invasion to predict outcome after resection in patients with hepatocellular carcinoma. Gastroenterology 137: $850-855$ 
18. Cha DI, Jang KM, Kim SH, Kim YK, Kim H, Ahn SH (2020) Preoperative prediction for early recurrence can be as accurate as postoperative assessment in single hepatocellular carcinoma patients. Korean J Radiol 21:402-412

19. Zhang L, Kuang S, Chen J et al (2019) The role of preoperative dynamic contrast-enhanced 3.0-T MR imaging in predicting early recurrence in patients with early-stage hepatocellular carcinomas after curative resection. Front Oncol 9:1336

20. Zhang Z, Jiang H, Chen J et al (2019) Hepatocellular carcinoma: radiomics nomogram on gadoxetic acid-enhanced MR imaging for early postoperative recurrence prediction. Cancer Imaging 19:22

21. Kim S, Shin J, Kim DY, Choi GH, Kim MJ, Choi JY (2019) Radiomics on gadoxetic acid-enhanced magnetic resonance imaging for prediction of postoperative early and late recurrence of single hepatocellular carcinoma. Clin Cancer Res 25:3847-3855

22. Hectors SJ, Lewis S, Besa C et al (2020) MRI radiomics features predict immuno-oncological characteristics of hepatocellular carcinoma. Eur Radiol 30:3759-3769

23. Qin X, Yang T, Huang Z et al (2019) Hepatocellular carcinoma grading and recurrence prediction using $T_{1}$ mapping on gadolinium-ethoxybenzyl diethylenetriamine pentaacetic acidenhanced magnetic resonance imaging. Oncol Lett 18:2322-2329

24. Kitao A, Zen Y, Matsui O, Gabata T, Nakanuma Y (2009) Hepatocarcinogenesis: multistep changes of drainage vessels at CT during arterial portography and hepatic arteriographyradiologic-pathologic correlation. Radiology 252:605-614

25. Choi JY, Lee JM, Sirlin CB (2014) CT and MR imaging diagnosis and staging of hepatocellular carcinoma: part I. Development, growth, and spread: key pathologic and imaging aspects. Radiology 272:635-654
26. Matsui O, Kobayashi S, Sanada J et al (2011) Hepatocellular nodules in liver cirrhosis: hemodynamic evaluation (angiographyassisted CT) with special reference to multi-step hepatocarcinogenesis. Abdom Imaging 36:264-272

27. Choi JY, Lee JM, Sirlin CB (2014) CT and MR imaging diagnosis and staging of hepatocellular carcinoma: part II. Extracellular agents, hepatobiliary agents, and ancillary imaging features. Radiology 273:30-50

28. Zhang Y, Kuang S, Shan Q et al (2019) Can IVIM help predict HCC recurrence after hepatectomy? Eur Radiol 29:5791-5803

29. Marrero JA, Kulik LM, Sirlin CB et al (2018) Diagnosis, staging, and management of hepatocellular carcinoma: 2018 practice guidance by the American Association for the Study of Liver Diseases. Hepatology 68:723-750

30. Lee EC, Kim SH, Park H, Lee SD, Lee SA, Park SJ (2017) Survival analysis after liver resection for hepatocellular carcinoma: a consecutive cohort of 1002 patients. J Gastroenterol Hepatol 32:10551063

31. Cheng ZJ, Yang PH, Qu SP et al (2015) Risk factors and management for early and late intrahepatic recurrence of solitary hepatocellular carcinoma after curative resection. HPB (Oxford) 17:422427

32. Van der Pol CB, Lim CS, Sirlin CB et al (2019) Accuracy of the liver imaging reporting and data system in computed tomography and magnetic resonance image analysis of hepatocellular carcinoma or overall malignancy — a systematic review. Gastroenterology 156 : 976-986

Publisher's note Springer Nature remains neutral with regard to jurisdictional claims in published maps and institutional affiliations. 